ZWP grating diffraction imaging
instrument and its application in
optics experimental courses teaching

Weiping Zhang, Yuyang Huang, Yaling Tang, Yulu Zhou

Weiping Zhang, Yuyang Huang, Yaling Tang, Yulu Zhou, "ZWP grating diffraction imaging instrument and its application in optics experimental courses teaching," Proc. SPIE 9793, Education and Training in Optics and Photonics: ETOP 2015, 97931H (8 October 2015); doi: 10.1117/12.2223113

Event: Education and Training in Optics and Photonics: ETOP 2015, 2015, Bordeaux, France 


\title{
ZWP Grating Diffraction Imaging Instrument and its application in optics experimental courses teaching
}

\author{
Weiping Zhang*, Yuyang Huang, Yaling Tang, Yulu Zhou \\ College of Physical Science and Technology, Guangxi University, Nanning, 530004, PR China
}

\begin{abstract}
Based on the spectral combination function of grating and the bi-grating diffraction imaging effect, ZWP Grating Diffraction Imaging Instrument has been designed and developed, and some new experiment courses about grating diffraction have been set up for college students using the instrument. The new grating experiments are aimed to help students understand the comprehensive knowledge of grating diffraction. Also, it is a good training to improve student's hands-on abilities. The instrument has been used by more and more universities.
\end{abstract}

Key words: bi-grating imaging, diffraction imaging, experimental teaching, optics experimental courses

\section{INTRODUCTION}

The interference and diffraction of light is an important content of modern optical theoretical teaching and experimental teaching ${ }^{[1-3]}$. Grating is an important optical element and one of the typical content in optical teaching. It has not only function of dispersion and separation of light, but also function of diffraction imaging ${ }^{[4]}$. However, for a long time, the traditional teaching of grating diffraction has one-sidedness, which introduces the optic splitting and dispersion of grating, but the combination spectrum imaging effect and the bi-grating diffraction imaging effect are not included $^{[5,6]}$.

In order to overcome the shortage of diffraction grating teaching and let the students comprehensively understand the effect of diffraction grating, a bi-grating diffraction imaging instrument based on the bi-grating dispersioncombination spectral imaging effect was designed by us ${ }^{[7,8]}$. Through this instrument, "Barrier around the concept of objects" can be achieved. Based on this instrument, two new kinds of diffraction grating imaging experiments for students were set up: one is the observation of bi-grating diffraction imaging effect and the related parameter measurement, another is the measuring the position of bi-grating diffraction image of the object. Also, the new content displaying Fresnel hologram with a white light source was added to the existing holography experiments. Now, these new experiments are open in more and more universities. The new experimental instrument has been produced by specialized manufacturers, which is named ZWP Grating Diffraction Imaging Instrument.

\section{BI-GRATING DISPERSION - COMBINATION SPECTRAL IMAGING EFFECT}

In 2001, the new effect of the grating diffraction was reported by us, which is the spectral combination imaging effect, and bi-grating dispersion - combination spectral imaging effect. The multicolor light, arranged according to the spectra at different incident angle into the grating, get beam of the same (or similar) outgoing angle after the diffraction of grating, namely, the combination spectrum effect, which is the opposite of grating dispersion effect. Grating combination spectrum imaging means a clear image of object can be formed by using the spectral combination function of a grating.

*zwp2001@126.com 


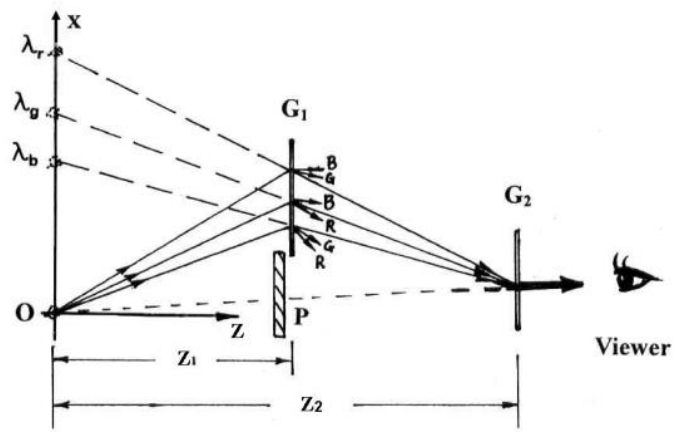

Fig.1 The schematic diagram of the light path of bi-grating diffraction imaging

Therefore, as shown in figure 1, the different wavelength of light beam (comes from the object) forms dispersion spectrum after the first grating, and when some order spectrum of light satisfy the grating spectral combination conditions, images of the objects can be seen behind the second grating. This effect is what we said bi-grating dispersion - combination spectral imaging effect, we also call it the bi-grating imaging effect. With this instrument, the object which is blocked off obstacles can be seen clearly (figure 2 ).

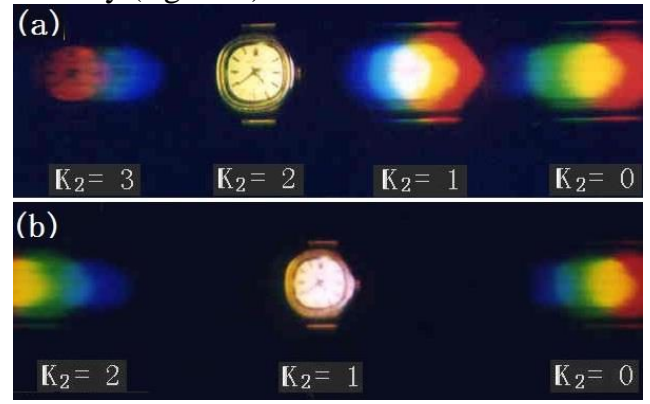

Fig.2 The image photos with the bi-grating diffraction imaging system

In figure 1 , the $\mathrm{G}_{1}$ and $\mathrm{G}_{2}$ are two unslanted plane transmission gratings, The $\mathrm{O}$ is the observed object, and $\mathrm{P}$ is a blocking plate. The function of grating $G_{1}$ is to make the light disperse to various objects spectrum from the object $O$. The grating $\mathrm{G}_{2}$ is located at the middle of the $\mathrm{G}_{1}$ 's diffraction $\mathrm{k} 1$ order beam where the combination spectra condition is satisfied, thereby the bi-grating imaging effect appears. At this time, according to our study, the relationship between the spatial frequencies, the diffraction order and the position of two gratings satisfy the following equation:

$$
\frac{k_{1} z_{1}}{d_{1}}=-w \frac{k_{2} z_{2}}{d_{2}}
$$

In the equation (1), $k_{1}$ is the diffraction order and $1 / d_{1}$ is the spatial frequency of $\mathrm{G}_{1}$, respectively. The $z_{1}$ represents the perpendicular distance between the grating $\mathrm{G}_{1}$ and the object $\mathrm{O}$. The $k_{2}$ is the diffraction order and $1 / d_{2}$ is the spatial frequency of $\mathrm{G}_{2}$. The $z_{2}$ represents the perpendicular distance between the grating $\mathrm{G}_{2}$ and the object $\mathrm{O}$. The negative sign in the equation indicates that the signs of the diffraction orders diffracted the gratings $G_{1}$ and $G_{2}$ are opposite. When the two gratings are parallel, the coefficient $\mathrm{w}$ is absolutely equal to 1 .

The features of bi-grating imaging system are as follows: 1) The system is a new type of diffraction imaging system. 2) "Barrier around the concept of objects can be successfully realized by the system. 3) The image is upright and virtual. 4) The object just needs an ordinary illumination (not only the monochromatic light). The difficulty of the content of spectral combination effect of a grating and the bi-grating dispersion - combination spectral imaging effect is moderate, which is suitable to introduce into the physical optics experiment in universities. These experiments not only let students observe the typical diffraction phenomena, but also let them understand the nature of grating diffraction both from dispersion and combination spectrum aspects.

\section{ZWP GRATING DIFFRACTION IMAGING INSTRUMENT}

The new experiment needs new equipment. As shown in figure 1, the positions of the light source and two gratings are not in one line, but the existing light holder cannot meet this requirement. Thus the ZWP grating diffraction imaging 
instrument was designed by us, which can be used in the new grating experiments. This instrument consists of three parts (figure3).

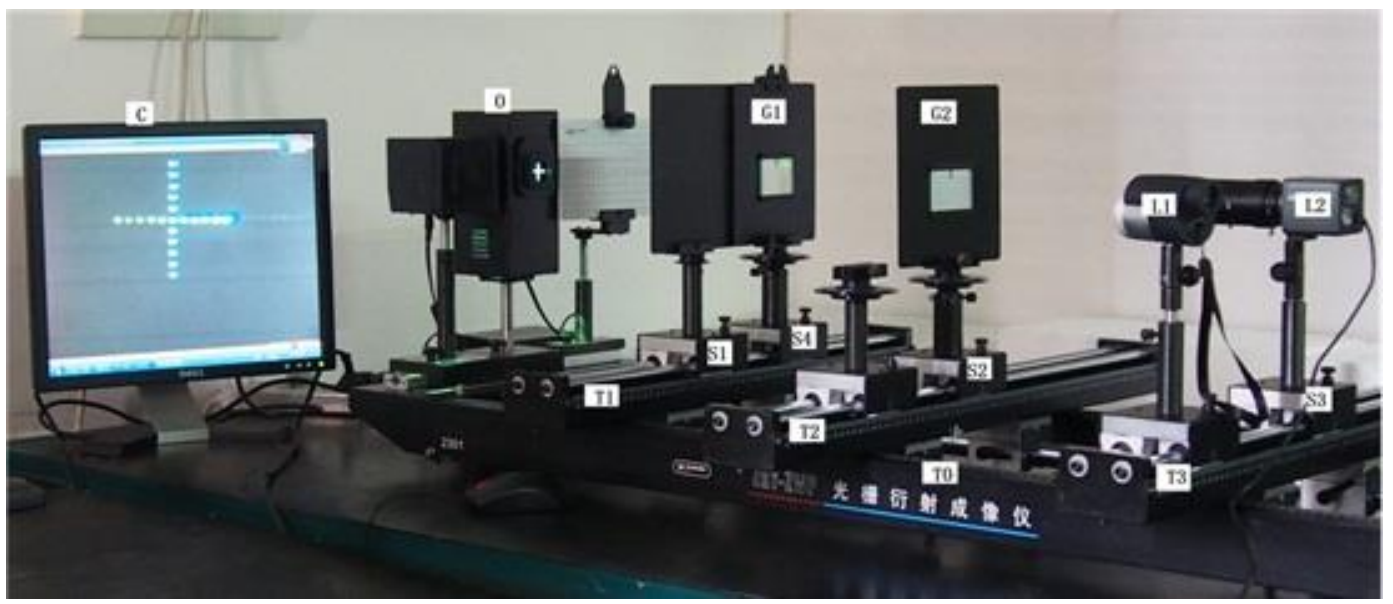

Fig.3 ZWP Grating Diffraction Imaging Instrument.

1) The mechanical moving part. It is mainly comprised of the pedestal, the track $T_{0}$ in $Z$ direction, the tracks $T_{1}, T_{2}$ and $\mathrm{T}_{3}$ in $\mathrm{X}$ direction and the slide brackets $\mathrm{S}_{1}, \mathrm{~S}_{2}, \mathrm{~S}_{3}$ and $\mathrm{S}_{4}$.

The two tracks in the $\mathrm{X}$ direction can slide in the track of $\mathrm{Z}$ direction. Each slide block and bracket above slide block can slide in the track of $\mathrm{X}$ direction, so the sliders can be moved on the desktop in the $\mathrm{X}-\mathrm{Z}$ plane within the track length range. Thus the optical elements supported by the slide block will also be able to move on the desktop. There are rulers on the track, so we can know the coordinates of the optical elements.

2) The optical elements part. It contains the light source $O$, the diffraction grating $G_{1}$ and combination grating $G_{2}$ and the telescope $\mathrm{L}_{1}$.

Four diffraction gratings with different spatial frequencies plane are provided to fit different combinations of grating diffraction imaging experiments. In order to observe diffraction image conveniently, the instrument is equipped with a telescope. As we need, the image can be observed through a telescope which is placed on slide 4 .

3) The computer image acquisition system. It consists of a computer, the camera L2 and the image reading system.

The computer image acquisition system can shoot the observed image and stored in the computer for subsequent process.

\section{SET UP EXPERIMENT BY USING ZWP GRATING DIFFRACTION IMAGING INSTRUMENT}

The experiment based on ZWP grating diffraction imaging instrument is not a new experiment but a type of diffraction experiments ${ }^{[9-11]}$. It contents several experiments including the observation of the grating diffraction effect, the bi-grating imaging effect and law, and displaying Fresnel hologram with a white light source. The serial experiments( of observation, verification and design) let students observe the typical diffraction phenomena of grating dispersion and combination, observe the grating combined spectrum effect and the bi-grating diffraction imaging effect, verify the grating equation and the bi-grating diffraction imaging law. The displaying Fresnel hologram with a white light source should be designed for an experiment, which is helpful to develop students' thinking.

We set up the following experiments:

Experiment one: Observation of bi-grating diffraction imaging and testing the relative parameter ${ }^{[12]}$.

In this verification experiment, the students are asked to adjust the ZWP grating diffraction instrument, and observe the bi-grating dispersion - combination spectral imaging effect. The students should use different combinations of two gratings with different parameters to verify the grating equation, as well as measure and calculate the spatial frequency of an unknown grating.

Experiment two: Measuring the virtual image position formed by the bi-grating system.

In this comprehensive experiment, the students are asked to adjust the bi-grating diffraction imaging instrument with the different angle $\theta$ between the two gratings, then use the camera to shoot the combined virtual images after the bi-grating and the original image of the object. By using the computer program to calculate the lateral offset between the 
virtual and original images, the students should draw the relationship diagrams between the lateral offset and the position and orientation of the second grating.

Experiment three: Displaying Fresnel hologram with a white light source.

If one of the two gratings in the bi-grating system above is replaced by a Fresnel hologram with a spatial frequency similar with the original one, the Fresnel hologram can be displayed with a white light source ${ }^{[13]}$. Therefore, we apply the ZWP grating diffraction imaging instrument to the existing holographic experiments, and add the new content of displaying Fresnel hologram with a white light source. According to this new experiment, the students can observe the colorful hologram image of original object. This new experiment can enhance the interest of hologram experiment for student, and enrich and improve the original holographic experiment.

A designing experiment is set up with its content as follows: design and shoot a hologram whose central spatial frequency is $300-5001 / \mathrm{mm}$, and place a auxiliary grating whose spatial frequency is $1000 \mathrm{l} / \mathrm{mm}$ and a white light in the bi-grating diffraction imaging instrument. The hologram with the color needed can be displayed. This experiment requires students to understand more deeply about the principle of the hologram displayed, know the main problem to be solved in displaying Fresnel hologram with a white light source, as well as learn to use the grating in such experiment.

When using ZWP grating diffraction instrument to perform the above experiments, the students learn more knowledge about grating, and their handling ability can be trained. The instrument is designed with lateral and perpendicular tracks, but the gratings moving along diagonal lines since the optical path is " $Z$ " shape. The student should move the grating in lateral and perpendicular directions at the same time, which is good for training their coordinate abilities.

In the second experiment above, the students not only need to observe the bi-grating dispersion - combination spectral imaging effect with different angle $\theta$ between the two gratings by adjusting ZWP grating diffraction instrument, but also need to use the computer to capture images and do data processing. This is a comprehensive training for students.

Since 2009, 8 sets of ZWP grating diffraction instrument have been employed in new experimental courses in our university. Fig 4 showed our college students doing experiments. The new experiments have been praised by students and peer experts in China. Currently, the instrument and our new experiments have been extended to the other universities.

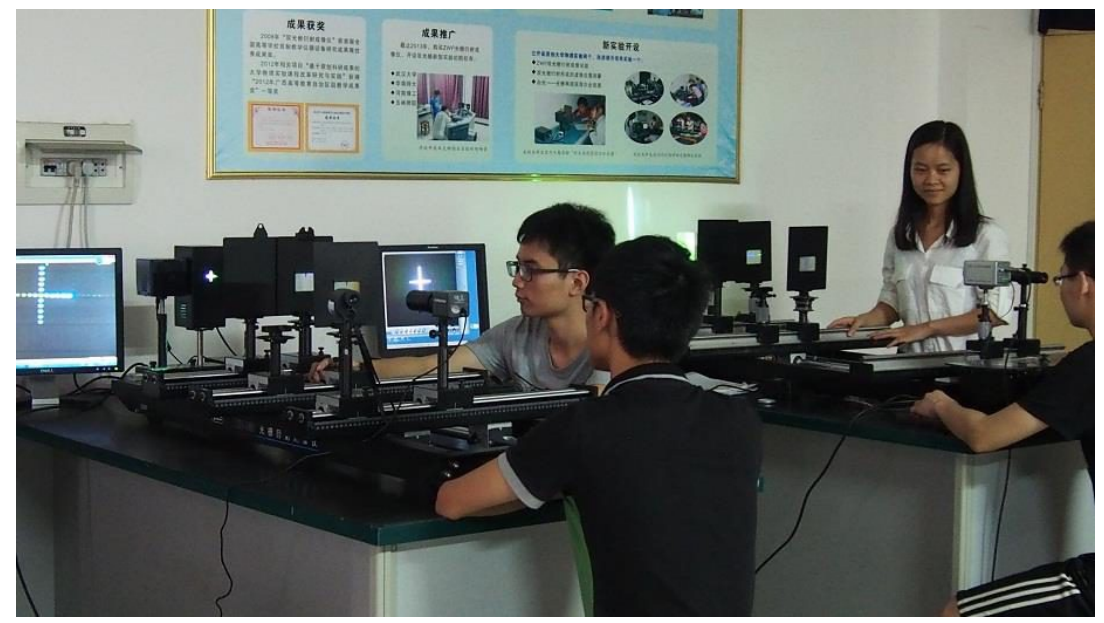

Fig.4 College students doing the experiments.

\section{CONCLUSION}

ZWP grating diffraction imaging instrument is specially designed to observe and study bi-grating diffraction imaging effect, which can well support to open the new experimental courses about gratings diffraction imaging. The new diffraction experiments based on ZWP diffraction grating imaging instrument provide a wide range of training for students. According to the new experiments, students can have a comprehensive understanding on the diffraction characteristics (including the dispersion and combination spectrum effects) of grating.

The new grating experiments not only involve the basic grating diffraction characteristics, but also include new physical phenomenon and extensive knowledge of physical optics. It can increase students' understanding of the light 
diffraction, as well as develop their innovation. So the instrument and the new experiments are worthy of promotion in colleges and universities.

\section{ACKNOWLEDGMENTS}

The authors acknowledge financial support from the Chinese National Natural Science Foundation (No. 61167001) and Guangxi University Foundation (No. 20140404) and the Education Development Foundation of Guangxi (No. 2015JGA138)

\section{REFERENCES}

[1]Paul W. Zitzewitz, Ph.D. Physics Principles and Problems. McGraw-Hill Companies(2004).

[2]Hecht E, Zajac A. Optics. Massachusetts: Addison-Wesley(2003).

[3]Glazebrook, Physical Optics: Textbooks of Science. Wexford College Press(2002).

[4]Dong, J. and Zhang, W.P., "Imaging of virtual objects with a plane periodic grating", Opt. Lett., 34(20), 3232-3234 (2009).

[5]Zhang,W.P.and Wei,W.L. ,"Method for spectrum imaging”. Proc. SPIE 4548:99-102(2001).

[6]Zhang,W.P.and He,X.R., "The spectral combination characteristic of grating and the bi-grating diffraction imaging effect "[J]. Science in China Series G: Physics Mechanics and Astronomy, 50(1): 1672-1799(2007).

[7]Huang,C.G., Zhang,W.P., Ma,X.M., Huang,Y.Y.and Zhou,W.Z.,"Development of bi-grating diffraction imaging apparatus and its usage", Experimental Technology and Management,Vol.28 No.9Sep(2011).

[8]Zhang,W.P., "Experiment Apparatus of Diffraction Imaging","Patent No:02290557.X.

[9] Zhang W.P., Huang G.1., Xiao Y.F., Luo T.J., Huang C.g., Huang Y.Y.," Combining teaching with researches closely to create new grating experiments", Research and Exploration in Laboratory.Vol.30(3),256-259(2011)

[10]Zhang,W.P., Zhu,F., Wang,C.Z., Gao,Y.C.and Liu,L., "Factors of influencing the value of w in the bi-grating diffraction imaging equation" Proc. SPIE Vol. 8556 85561L-1(2012).

[11] Zhang,W.P., Liu,L., Sun,S.Q., Zeng,X.j.and Lu,Q,"Design of a high-accuracy bi-grating imaging instrument for non-dispersive imaging"Proc. SPIE Vol. 9046 90461G-2(2013).

[12]Wu, W.M., Wei, W.L., Deng, W. and Gao Y.J, "University physics experiment” [M], Beijing: Science Press, 2010, 272-274.

[13]Zhang,W.P., He, X.R.and Shen,X.M., "Principle of the ordinary transmission hologram displaying with a white light source ”,Journal of Guangxi University (Nat Sci Ed) Vol.28.No.4 Dec.(2003). 